\title{
A Survey of Chinese Medicinal Herbal Treatment for Chemotherapy-Induced Oral Mucositis
}

\author{
Gesa Meyer-Hamme, ${ }^{1}$ Kathrin Beckmann, ${ }^{1}$ Janine Radtke, ${ }^{1}$ Thomas Efferth, \\ Henry Johannes Greten, ${ }^{3}$ Matthias Rostock, ${ }^{4,5}$ and Sven Schröder ${ }^{1,3}$ \\ ${ }^{1}$ HanseMerkur Center for Traditional Chinese Medicine at the University Medical Center Hamburg-Eppendorf, \\ Martinistraße 52, 20246 Hamburg, Germany \\ ${ }^{2}$ Department of Pharmaceutical Biology, Institute of Pharmacy and Biochemistry, Johannes Gutenberg University, \\ Staudinger Weg 5, 55128 Mainz, Germany \\ ${ }^{3}$ ICBAS, University of Porto, Rua de Jorge Viterbo Ferreira No. 228, 4050-313 Porto, Portugal \\ ${ }^{4}$ Institute of Complementary Medicine, University Hospital Zurich, 8006 Zurich, Switzerland \\ ${ }^{5}$ Hubertus Wald Tumorzentrum, University Cancer Center Hamburg, University Medical Center Hamburg-Eppendorf, \\ Martinistraße 52, 20246 Hamburg, Germany
}

Correspondence should be addressed to Gesa Meyer-Hamme; meyer-hamme@tcm-am-uke.de

Received 9 May 2013; Accepted 21 August 2013

Academic Editor: Sookyung Lee

Copyright (C) 2013 Gesa Meyer-Hamme et al. This is an open access article distributed under the Creative Commons Attribution License, which permits unrestricted use, distribution, and reproduction in any medium, provided the original work is properly cited.

Oral mucositis is one of the common side effects of chemotherapy treatment with potentially severe implications. Despite several treatment approaches by conventional and complementary western medicine, the therapeutic outcome is often not satisfactory. Traditional Chinese Medicine (TCM) offers empirical herbal formulas for the treatment of oral ulceration which are used in adaptation to chemotherapy-induced mucositis. While standard concepts for TCM treatment do not exist and acceptance by conventional oncologists is still low, we conducted a review to examine the evidence of Chinese herbal treatment in oral mucositis. Eighteen relevant studies on 4 single herbs, 2 combinations of 2 herbs, and 11 multiherbal prescriptions involving 3 or more compounds were included. Corresponding molecular mechanisms were investigated. The knowledge about detailed herbal mechanisms, especially in multi-herbal prescriptions is still limited. The quality of clinical trials needs further improvement. Metaanalysis on the existent database is not possible but molecular findings on Chinese medicinal herbs indicate that further research is still promising for the treatment of chemotherapy-induced oral mucositis.

\section{Introduction}

Oral mucositis is one of the most common side effects of chemotherapy treatment with potentially severe implications. According to the American National Cancer Institute, ulcerative oral mucositis occurs in approximately $40 \%$ of patients receiving standard-dose chemotherapy [1]. Medical interventions are required in about $50 \%$ of these patients, including changes of medication or chemotherapy dose reduction. Severe mucositis symptoms occur in up to $80 \%$ in high dose chemotherapy treatments of leukaemia or in stem cell transplant regimens [1].
Mucosal damages may be induced for example, by antimetabolites such as methotrexate, 5-fluorouracil, anthracyclines such as doxorubicin and bleomycin, alkylating antineoplastic agents such as cyclophosphamide and busulfan, taxanes and the platinum complexes, including cisplatin and carboplatin $[1,2]$. All of them may have possible toxic effects on rapidly dividing mucosal cells, partly related to drug secretion in the saliva. Saliva volume and consistence as well as the oral microbial flora may be altered, affecting the mucosal metabolism [3]. Several molecular mechanisms are involved in the pathogenesis of mucositis, such as oxidation and apoptosis mediated by nitric oxide $(\mathrm{NO})$, cyclooxygenase 
(COX), protein kinases, cytokines, and nuclear factors [4]. The research field involves also genetic-based risk factors [5]. Epigenetic changes of DNA methylation are discussed as being responsible for inflammatorial precancerous conditions [6]. A cancer diagnosis itself may lead to posttraumatic stress disorder (PTSD), causing depression and anxiety as well as an increased level of biomarker expression, such as interleukin-6 (IL-6), tumor necrosis factor- $\alpha$ (TNF- $\alpha$ ), cortisol, and high-reactive sensitive C-reactive protein. The incidence of oral ulceration was associated with the level of PTSD. These effects were observed to be significantly higher in malignant than in benign diagnosed breast tumor patients [7].

Typical manifestations of oral mucositis are soreness, edema, erythema, ulcerations, bleeding, pain, difficulties in swallowing and possible alteration of taste, and they may severely affect the patient's quality of life. Impaired nutrition and complications by viral, bacterial, or mycotic infections may additionally increase the risk of anticancer treatment delay [8]. Mucositis grading is based on clinical aspects and the nutritional state, as seen in Table 1. Grades III and IV are considered as severe mucositis $[9,10]$ and in more than onethird of these patients the next chemotherapy cycle needs to be delayed leading to a possible deterioration of treatment [11].

When uncomplicated by infections, mucositis may be self-limiting in about 2 to 4 weeks [1]. Mucosal damages and local factors such as periodontitis and suboptimal oral hygiene increase the risk of infections. Systemic exacerbation is facilitated by the commonly decreased immunological status $[12,13]$. Consequences are an increased mortality risk as well as a prolonged hospitalisation, including the necessity of fluid replacement and parenteral nutrition, causing an increase of costs [9].

1.1. Preventive and Treatment Methods by Conventional Medicine. The detailed guidelines for prevention and treatment of mucositis are depending on the chemotherapy regimens used in each case. General prevention instructions include prior dental examinations and treatment and optimal oral care $[10,16]$, as well as avoidance of spicy, hard and hot foods and saline-peroxide mouthwashes [16].

There are numerous western experimental preventive and therapeutical interventions for oral mucositis. Updated clinical practice guidelines for the prevention and treatment of mucositis were published by the Mucositis Study Section of the Multinational Association of Supportive Care in Cancer and the International Society for Oral Oncology (MASCC/ISOO) in 2007 [10], suggesting the use of keratinocyte growth factor-1 (KGF-1) for preventing mucositis in high-dose chemotherapy regimens. Cryotherapy was suggested for melphalan, 5-fluorouracil, and etidronate. Systemic glutamine was not recommended because of severe toxicity. Mouthwashing with granulocyte-macrophage-colony stimulating factor (GM-CSF) did not show consistent effect. Updated results are frequently published by MASCC/ISOO [17]. According to a Cochrane review from 2010, nine interventions for prevention and treatment of mucositis showed statistical benefit: allopurinol, amifostine, cryotherapy, intravenous glutamine, honey, KGF-1, laser, aloe vera, and polymixin/tobramycin/amphotericin (PTA) antibiotic pastille or paste compared to either placebo or no treatment [18]. The updated Cochrane review, published in February 2013, came to the conclusion that cryotherapy and keratinocyte growth factor had some benefits in preventing mucositis and sucralfate showed effects in reducing the severity of mucositis. Aloe vera, amifostine, granulocyte growth factor, honey, laser, and PTA did not show consistent effects [19].

Even concerning the progress achieved during the last years, chemotherapy-induced oral mucositis continues to be a challenge for a positive cancer treatment outcome $[2,18$, 19]. The development of further treatment options for oral mucositis remains an important research objective.

1.2. Complementary Medicine with Western Herbs. In western complementary medicine several herbal treatment approaches are existent, including Salvia officinalis, Camomilla matriciana, Calendula officinalis, Hamamelis virginiana, Tormentilla rhizome, Commiphora molmol, Rhataniae radix, Myrtilli fructus, Althaea, Malva, Cetraria islandica, Linum usitatissimum, Caryophylli flos, Hippophea rhamnoides, Aloe vera, Carica papaya, Centaurii herba, Gentianae radix, Menyanthis folium, Eriodictyon crassifolium, Oleum olivae, and Citrus limon. They are applied as single infusions for gargling or topical application [2]. Of these, Salvia officinalis, Chammomilla matriciana, Aloe vera, and Gentianae radix have also been used in the tradition of TCM.

Up to $80 \%$ of cancer patients use some kinds of Complementary and Alternative Medicine (CAM) therapies to support their conventional cancer treatments [20, 21]. Herbal treatment is the most frequently used CAM therapy and many of the used herbs originate from TCM [22, 23]. TCM offers empirical herbal formulas for treating mouth ulcers and stomatitis which have frequently been used in complementary treatment of oral mucositis in the last decades, but the evidence of these therapies is unclear. While standard concepts for this kind of treatment do not exist and acceptance by conventional oncologists is still low, we conducted this review to critically examine the evidence of Chinese herbal treatment in oral mucositis.

\section{Methods}

2.1. Objective. The objective of this article is to examine the role of Chinese herbal medicine approaches to oral mucositis in search of adjuvant treatment options for minimizing a painful and risky side effect of chemotherapy as a potential cooperation of western and Chinese medicine.

2.2. Search Strategy and Selection Criteria. Electronic searches of PubMed, MEDLINE via OVID, EMBASE via OVID, Cochrane Database, CNKI and reference lists of relevant articles were undertaken. The mesh-terms used were chemotherapy, chemotherapy-induced, oral ulcer, mouth ulcer, oral mucositis, stomatitis, Chinese herbal 
TABLE 1: WHO oral toxicity scale $[14,15]$.

\begin{tabular}{lcccc}
\hline Grade 0 & Grade I & Grade II & Grade III & Grade IV \\
\hline (None) & (Mild) & (Moderate) & (Severe) & (Life-threatening) \\
None & Oral soreness, erythema & Oral erythema, ulcers, solid diet tolerated & Oral ulcers, liquid diet only & Oral alimentation impossible \\
\hline
\end{tabular}

medicine, medicinal herbs, Chinese herbs, traditional Chinese medicine, antioxidant, anti-inflammatory. All interventional clinical trials concerning chemotherapyinduced oral mucositis treated by Chinese herbal medicine that offered an English or Chinese abstract were reviewed. Articles investigating herbal or animal products used in the tradition of Chinese medicine were included. Articles investigating radiochemotherapy-induced oral mucositis were excluded, unless the chemotherapy-related results were separately processed.

2.3. Data Analysis. No meta-analysis was effected since there were reservations with regard to the high risk of bias due to inadequate study designs and a diversity of herbal formulas. We limited the discussion to the comparison of single herbs and herbal formulas and to the quality of studies which have to date been set up with regard to this topic.

\section{Results}

A total of 686 articles were retrieved from electronic searches and from examination of reference lists of clinical and review articles. After screening titles and/or abstracts, 632 articles were excluded since the focus was either on an intervention rather than on oral mucositis and Chinese herbal treatment or they were duplicated studies or not relevant. From a total of 54 articles which were retrieved for detailed evaluation, 18 studies representing 1,476 patients met the selection criteria and were included in the review, focusing on 4 single herbs, 2 combinations of 2 herbs, and 11 multi-herbal prescriptions involving 3 or more components. For a summary of the investigated studies, see Table 2 .

\section{Single Herbs or Single Herbal Compounds}

4.1. Evodiae fructus. In terms of TCM theory, Evodiae fructus has been used for nausea and pain caused by cold exposition [24-26]. Evodiamine, a major compound of Evodiae fructus, was found to inhibit inducible nitric oxide synthase (iNOS) and nuclear factor kappa-light-chain-enhancer of activated $\mathrm{B}$ cells $(\mathrm{NF}-\kappa \mathrm{B})$ activation, as well as the cyclo-oxygenase- 2 (COX-2) expression, hypoxia-inducible factor 1-alpha (HIF$1-\alpha$ ) accumulation and prostaglandin E-2 (PGE-2) synthesis, and interferon-gamma (INF- $\gamma$ ) mediated processes in murine 264.7 macrophage-like cell lines [27].

Application of Evodiae fructus extract on acupoint Yongquan (KID1) was reported to have positive effects on mucositis symptoms in $92 \%$ of chemotherapy patients in an uncontrolled case study $(n=50)$ measured by subjective scales [28]. Ulcer grading has not been mentioned [28]. The result requires further investigation with higher-quality study designs.

4.2. Rhodiola algida. Rhodiola subspecies are used as common tonics in various Asian regions. In Tibetan medicine, it has an ancient tradition for its antifatigue effects, as well as for cardiovascular diseases, pneumonia, and hemoptysis [29]. Rhodiola algida is externally used for injuries, burns, and scalds [29] and has shown immunomodulatory effects by interleukin 2 (IL-2) regulation in Th-1 cells and interleukin 4, 6 , and 10 (IL-4, IL-6, IL-10) regulation in Th-2 cells [30]. In a randomised, controlled, two-armed clinical study on breast cancer patients ( $n=130$; treatment: $n=65$; control: $n=65)$ [31], oral administration of Rhodiola algida resulted in faster recovery of white blood cell count as well as fewer and smaller ulcers compared to the non-treatment control group, judged by clinical measurement. Furthermore, positive effects of Rhodiola algida on lymphocyte proliferation was reported while animal feeding with Rhodiola algida did not show toxic effects [31]. These findings are promising, but so far no further trials confirming the results have been found.

4.3. Catechu from Acacia catechu. Catechu is an extract of Acacia catechu which is clinically used for tissue regeneration, wound healing, sores and abscesses as well as mouthwash for oral ulcers [26, 32]. In combination with Scutellariae baicalensis radix, anti-inflammatory effects are reported in animal and human immortalized cell lines [33]. Local application of Catechu powder had a superior effect on oral mucositis compared to local norfloxacin application in a randomized, controlled, two-armed clinical study on chemotherapy patients $(n=60$; treatment: $n=30$; control: $n=30)$, judged by clinical measurement [34]. Amelioration occurred in all patients (100\%) treated with Catechu and in $73.3 \%$ of the norfloxacin group. Further trials confirming this result have not been found.

4.4. Kangfuxin from Periplaneta americana. Kangfuxin is an ethanolic extract of Periplaneta americana, used for its anti-inflammatory and wound healing qualities in ulcerative and inflammatory diseases, including recurrent aphthous ulcers [35]. Regulating effects of the cytokine expression IL4 , interleukin 5 (IL-5), IFN- $\gamma$, and TNF- $\alpha$ by decreased gene expression have been reported [36]. In a randomized, controlled, two-armed clinical study on chemotherapy patients ( $n=64$; treatment: $n=26$; control: $n=38$ ), topical Kangfuxin application showed an oral mucositis rate of $36 \%$, compared to saline gargling with a mucositis rate of $84 \%$ [37]. This result as well requires verification by more trials with high-quality study designs. 


\section{Herbal Combinations}

5.1. Lonicerae flos Plus Glycyrrhizae radix. Lonicerae flos and Glycyrrhizae radix are two important ingredients of the traditional herbal formula Yin Qiao San (Chinese) (Honeysuckle and Forsythiae Powder (English), Pulvis Lonicerae et Forsythiae (Latin)) from Wen Bing Tiao Bian (Systematic Differentiation of Warm Diseases) [38]. It is used in inflammatory conditions including those of skin and mucosa [39, 40]. In TCM tradition, Lonicerae flos is used for abscesses, swelling, ulcers and erysipelas [24-26]. It offers antioxidant properties, suppressing interleukin 1-beta (IL-1 $\beta$ ), IL-6, and COX-2 gene expression in human umbilical vein cells [41]. In traditional treatment, Glycyrrhizae radix is known to be useful for abscesses, scores, and ulcer treatment [24-26]. Anti-inflammatory effects are transmitted by variant compounds. Glycerol inhibits lipopolysaccharide (LPS)-induced NF- $\kappa$ B, IL-1, and IL- 6 mRNA activation, while liquiritigenin was found to inhibit the activation of NF- $\kappa \mathrm{B}$ in macrophages, decreasing iNOS and proinflammatory cytokines by inhibition of LPS-induced NF- $\kappa$ B DNA binding activity $[42,43]$.

This herbal combination has been investigated by two authors. In one randomized, controlled, two-armed clinical trial, the combined topical and internal use was compared to borax mouth washing in chemotherapy patients $(n=190$; treatment: $n=97$; control: $n=93$ ). Mucositis symptoms were found in $19.4 \%$ of treatment group patients and in $85.2 \%$ of control group patients, measured by the WHO scale for mucositis [44]. The study did not establish a control for the internal use of herbal medicine, comparing a dualmode treatment to a single control. In another clinical trial on chemotherapy patients ( $n=86$; treatment: $n=43$; control: $n=43$ ), herbal gargling solution was compared to hydrogen peroxide mouth washing [45]. Positive effects on mucositis symptoms were found in $95.3 \%$ of the herbal treatment group and $76.7 \%$ of the control group, measured by a subjective scale. Further investigation by high-quality studies is required.

5.2. Lonicerae flos Plus Glycyrrhizae radix Plus Astragali membranacei radix. In this formula, Astragali membranacei radix is used in addition to the herbs described above, Lonicerae flos and Glycyrrhizae radix. In traditional use, Astragali membranacei radix clears toxicity and is used for abscesses and promoting skin regeneration [24-26]. It has anti-inflammatory and immunoregulatory effects due to IL-2 and IFN- $\gamma$ release and IL-4 and iNOS suppression [46].

Topical use of this triple combination on oral mucositis was effected in a randomized, controlled, two-armed clinical trial ( $n=97$; treatment: $n=50$; control: $n=47$ ), with positive results compared to Dobell's solution (sodium borate, sodium bicarbonate, phenol and glycerol) [47]. The herbal treatment group showed an overall lower grade of ulcers. $30 \%$ did not show any mucositis symptoms while only $2 \%$ suffered from severe ulcers. In the Dobell's solution control group, $5 \%$ had remained without ulcers while $21.3 \%$ suffered from severe ulcers. There was no statistical difference in the duration of healing period. In addition to the investigated drugs, all patients received a basic treatment by intravenous injections of the Chinese herbal preparations Shengmai (Ginseng radix, Ophiopogonis radix, Schisandrae chinensis fructus), Shenqi Fuzheng (Codonopsis radix, Astragali membranacei radix), Ai Di (Acanthopanacis radix, Astragali membranacei radix, Ginseng radix) and Cantharidin, as well as vitamin supplements not further described, and tetracaine solution for local application. In total, this is a strongly tonifying herbal regime. Regarding this treatment by applying several intravenously administered herbs, significant conclusions on the investigated herbs of the gargle solution cannot be drawn due to various potential interactions between the single components.

5.3. Lonicerae flos Plus Ophiopogonis radix Plus Platycodonis radix. Lonicerae flos and Ophiopogonis radix are part of the classical herbal combination called Qing Ying Tang (Chinese), Clear the Ying Level Decoction (English), Decoctum Refrigerationis Qi Constructivi (Latin) from Wen Bing Tiao Bian (Systematic Differentiation of Warm Diseases) [38], which is composed by Rhinoceri cornu, Rehmanniae viridae radix, Scrophulariae radix, Lophatheri folium, Lonicerae flos, Forsythiae fructus, Coptidis rhizoma, Salviae miltiorrhizae radix and Ophiopogonis radix. It is used for certain forms of fever accompanied by a dry mouth [39, 40]. Ophiopogonis radix and Playcodonis radix are combined in Bai He Gu Jin Tang (Chinese), Lily Bulb Decoction To Preserve The Metal (English), Decoctum Firmans Metallum cum Lilio (Latin) from Yi Fang Yi Jie (Analytic Collection of Medical Formulas) [57], used for dry pharyngitis and bronchitis [39, 40]. Both formulas have other principal herbs whose effects are assisted by Lonicerae flos, Ophiopogonis radix and/or Platycodonis radix $[39,40]$.

In TCM theory, Ophiopogonis radix is used for sore throats and dry coughs [24-26]. There are scarce reports about single molecular mechanisms of Ophiopogonis radix. Opaw-2, a compound from Ophiopogonis radix, showed dose-dependent stimulation of lymphocyte proliferation in vitro [58]. Platycodonis radix had been traditionally used for relieving soreness, expelling pus and for treating general skin and mucosal diseases [24-26]. Platycodonis radix Saponins derived from Platycodonis radix showed anti-inflammatory effects via inhibition of COX-2, TNF- $\alpha$, and PGE2 expression, as well as reduction of inflammatory markers like the number of leukocytes and neutrophiles and edema [59].

For Lonicerae flos, see above.

This triple combination was investigated in a randomised, controlled, two-armed clinical trial on chemotherapy patients ( $n=65$; treatment: $n=30$; control: $n=35$ ). The treatment group patients received oral herbal administration while the control group patients used furacilin mouth washing and received oral administration of vitamins $\mathrm{B} 1$ and $\mathrm{C}$ and daily intravenous infusions of metronidazol. Basic treatment was performed by saline gargling accompanied by diet instructions. The herbal treatment group showed improvement of ulceration in $93 \%$, compared to $73.8 \%$ in the control group, classified by subjective scales of ulcer size [48]. In this trial, diversified control interventions impede the comparability to the treatment intervention. 


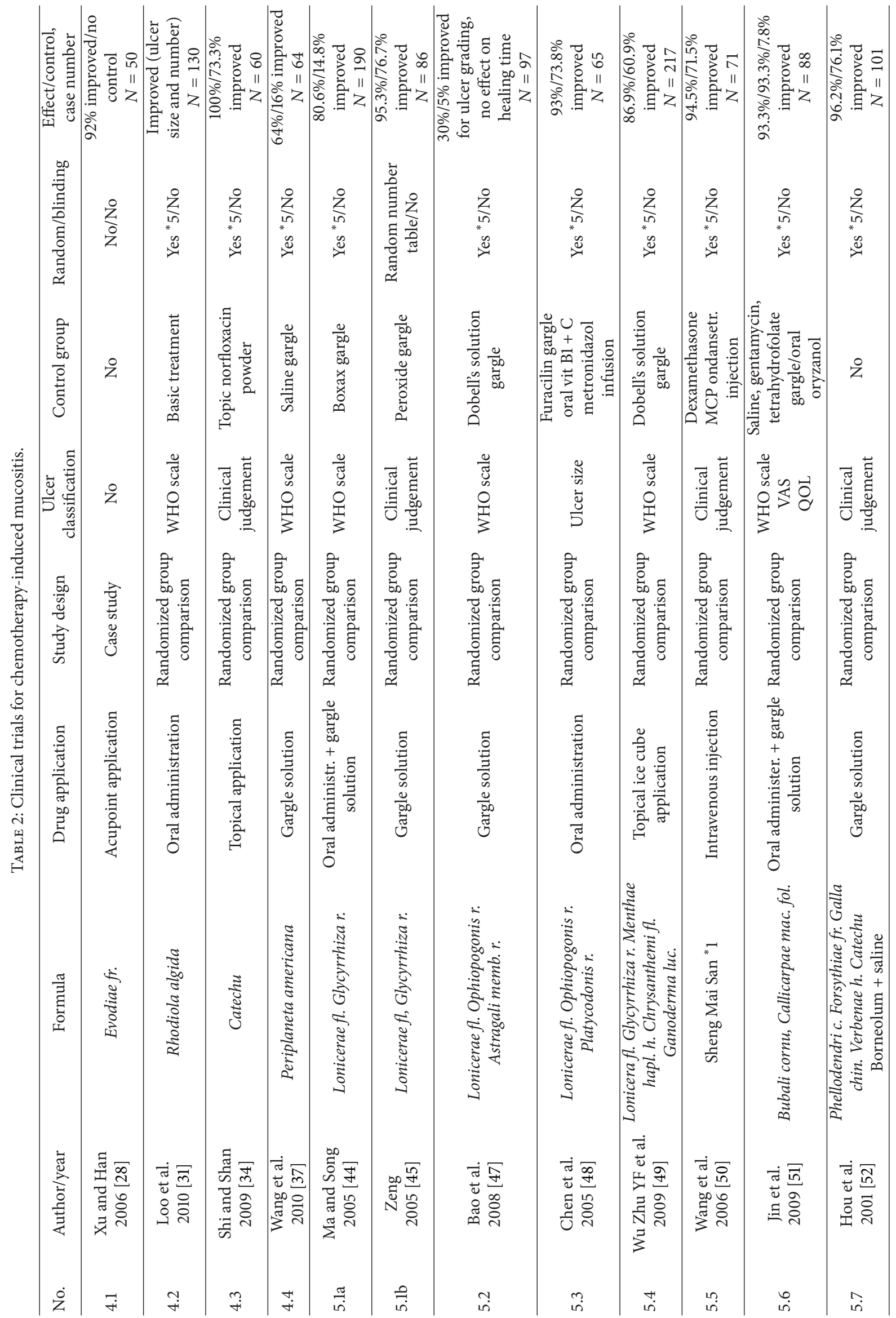




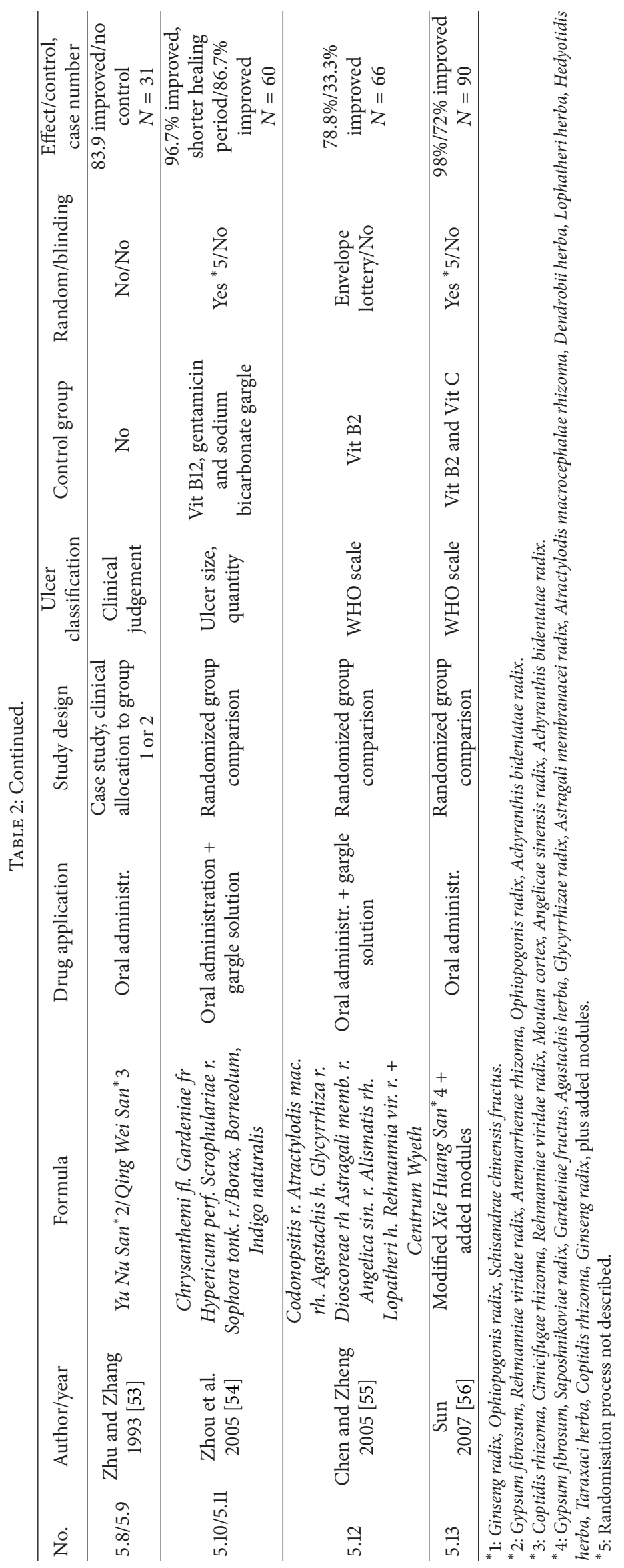


5.4. Lonicerae flos Plus Glycyrrhizae radix Plus Chrysanthemi flos Plus Ganoderma lucidum Plus Menthae haplocalycis herba. A Lonicerae flos, Glycyrrhizae radix and Menthae haplocalycis herba combination is found in Yin Qiao San $[39,40]$ as described above. A Chrysanthemi flos, Glycyrrhizae radix and Menthae haplocalycis herba combination is found in Sang Ju Yin (Chinese), Clear Wind Heat Tea (English)i Potio Mori et Chrysanthemi (Latin) from Wen Bing Tiao Bian [38] which contains Mori folium, Chrysanthemi flos, Armeniacae semen, Forsythiae fructus, Menthae haplocalycis herba, Platycodi radix, Glycyrrhizae radix, and Phragmitis rhizome and is used in beginning fever and coughing $[39,40]$. Ganoderma lucidum has not been found in these classical combinations.

In TCM theory, Chrysanthemi flos is used for septic wounds and abscesses [24-26]. Chrysanthemi flos compounds inhibit NO, PGE-2, TNF- $\alpha$, and IL- $1 \beta$ production, as well as iNOS and COX-2 expression in LPS-induced macrophages [60]. Ganoderma lucidum showed positive effects on intestinal epithelium healing [61] and refractory diabetic wounds [62] and was found to decrease NO, PGE2 , and proinflammatory cytokine production, including IL$1 \beta$, TNF- $\alpha$ and NF- $\kappa$ B in microglia [63]. Menthae haplocalycis herba in TCM theory is used for mouth sores, exanthema, and itching [24-26]. It showed antimicrobial activity against streptococcus mutans [64] and attenuated histamine release and PGD-2 synthesis in mast cells [65].

For Lonicerae flos and Glycyrrhizae radix, see above.

The decoction of Lonicerae flos, Glycyrrhizae radix, Ganoderma lucidum, Chrysanthemi flos, and Menthae haplocalycis herba was prepared for local ice cube application and compared to ambient tempered Dobell's gargle solution (sodium borate, sodium bicarbonate, phenol, and glycerol) in a randomised, controlled, two-armed clinical trial on gynaecological tumor patients receiving 5-FU chemotherapy ( $n=217$; treatment: $n=84$; control: $n=133$ ) [49]. The mucositis incidence resulted in $13.1 \%$ in the herbal treatment group and in $39.1 \%$ in the control group. The study design did not consider the fact that single ice application is known to have a preventive effect on oral mucositis in patients treated with 5-FU $[1,66]$, as there was no similar application design for both groups.

5.5. Shengmai San (Chinese), Generate the Pulse Powder (English). Shengmai San contains Ginseng radix, Ophiopogonis radix, and Schisandrae chinensis fructus and originates from Yu Xue Qi Yuan (Expounding on the Origins of Medicine) [67]. It is used in multiple clinical patterns including cardiovascular and neurologic disorders, diabetes, and cancer for its tonifying and yin nourishing properties [39, 40]. Regarding the single compounds, there are multiple reports about anti-inflammatory effects of ginsenosides by inhibition of proinflammatory cytokines and other mediators of inflammation including iNOS, NO, IF- $\gamma$, COX-2, NF- $\kappa$ B, and TNF- $\alpha$ [68]. Ginsenoside Rd showed wound healing effects on skin level, increasing the proliferation and migration of keratocyte progenitor cells and dermal fibroblasts by cyclic adenosine monophosphate (cAMP) induction via 9- $\beta$ $\mathrm{d}$-arabinofuranoside attenuation [69]. Schisandrae chinensis fructus in terms of TCM theory is astringent and preserving fluids [24-26]. Schisandrin B was found to inhibit ataxia telangiectasia and Rad3-related (ATR) protein kinase activity following DNA damage by inhibition of phosphorylation processes [70]. A hydrophobic fraction of dried Schisandrae chinensis fructus was found to suppress IL- $\beta$-induced NO and iNOS expression, as well as the transcription of IL-1 $\beta$ and inflammatory cytokines [71].

For Ophiopogonis radix, see above.

In a randomised, controlled, two-armed clinical trial ( $n=71$; treatment: $n=36$; control: $n=35$ ) on acute chemotherapy toxicity regarding primarily white blood cell and platelet counts as well as nausea, vomiting, and oral mucositis, intravenous Shengmai injection was compared to dexamethasone, metoclopramide, and ondansetron injection (which targeted nausea and vomiting rather than mucositis due to the ampler study design). Oral mucositis occurred in $5.5 \%$ of the treatment group and in $28.5 \%$ of the control group [50]. The control intervention of this trial was not specific for oral mucositis.

5.6. Bubali Cornu and Callicarpae macrophyllae folium. Bubali cornu is frequently found as modern substitute for Rhinoceri cornu (forbidden due to the Convention on International Trade in Endangered Species of Wild Fauna and Flora, also known as Washington Convention) in the classical formula Qing Ying Tang (see above). There are no classical formulas combining these two drugs, but this is a modern empirical combination called Shui Zhong Cao Tang Ji (Chinese), Water Grass Decoction (English). In terms of TCM theory, Bubali cornu has effects similar to Rhinoceri cornu, used for febrile diseases, exanthema, and convulsions [24-26]. It has shown antipyretic and antioxidant effects on proteins and inhibition of TNF- $\alpha$-induced PGE2 production, as well as protection against hydrogen peroxide $\left(\mathrm{H}_{2} \mathrm{O}_{2}\right)$-induced injuries in rat cerebral microvascular endothelial cells [72]. Callicarpae macrophyllae folium is reported to be used in TCM tradition for bleedings, hematoptysis, and hematemesis [26]. Anti-inflammatory, antimicrobial, and analgesic effects of Callicarpae macrophyllae folium are reported but not yet fully investigated [73].

This formula was topically and internally administered in a randomised, controlled, three-armed clinical trial $(n=$ 88; treatment: $n=30$; first control: $n=30$; second control: $n=28$ ) to chemotherapy patients, compared to topical application of gentamycin, tetrahydropholate, and saline gargling (first control) and to oral oryzanol administration (second control) [51]. There was a mean better outcome of curative voted cases from herbal treatment to the gentamycin/tetrahydropholate/saline control group (53.3\% to $50.0 \%)$. General improvement of oral mucositis symptoms was seen in $93.3 \%$ of both groups. Oral mucositis symptoms remained in $92.2 \%$ of the oryzanol group, showing an advantage of the two former regimens compared to oxyzanol administration alone. The comparison of the combined 
topical and internal use to a single control intervention does not allow significant conclusions.

5.7. Huang Wu Shu Kou Ye (Chinese), Yellow Five Decoction (English) (Phellodendri Chinensis Cortex, Forsythiae fructus, Verbenae officinalis herba, Borneolum, Galla chinensis, and Catechu). The name of this empirical prescription may be borrowed from Dang Gui Liu Huang Tang, Angelica Six Yellows Decoction (English), Decoctum Angelicae Sinensis et sex Luteorum (Latin) from Lan Shi Mi Cang (Sectrets from the Orchid Chamber) [74], as Phellodendri chinensis cortex is one of the contained 6 yellow coloured drugs (Rehmanniae viridae radix, Rehmanniae preparata radix, Coptidis rhizoma, Scutellariae baicalensis radix, Phellodendri chinensis cortex, and Astragali membranacei radix). The combination of Phellodendri chinensis cortex, Forsythiae fructus, Verbenae officinalis herba, Borneolum, Galla chinensis, and Catechu has not been found in classical prescriptions.

Phellodendri chinensis cortex is traditionally considered as anti-toxic and is used for abscesses and sores [24-26]. It was found to inhibit TNF- $\alpha$, IL- $1 \beta$, and iNOS production, as well as phosphorylation of extracellular-signal regulated kinases (ERK) and NF- $\kappa$ B activation in microglia cells [75]. Forsythiae fructus has a traditional use as anti-toxin as well as for erysipelas and abscesses [25-27]. A Forsythiae fructus compound, arctiin was found to decrease proinflammatory cytokine production including IL- $1 \beta$, IL- 6 , TNF- $\alpha$, and PGE2 , as well as NF- $\kappa \mathrm{B}$ and co-stimulating molecules such as peripheral membrane protein $\mathrm{B} 7-1$ and $\mathrm{B} 7-2$ in mouse leukaemic monocyte macrophage cell line (RAW 264.7) cells [76]. Verbenae officinalis herba in TCM terms removes toxicity and is used for sores and boils as well as pharyngitis $[26,77]$. Verbenae officinalis herba extractions were found to possess antioxidant, anti-inflammatory, and wound-healing properties [78]. Borneolum is a crystal steam distilled product of Cinnamomum camphora [26]. It is used externally for mouth sores, ulcerations, and wounds [24-26] and showed anti-inflammatory and antioxidant cell protective effects by decreasing iNOS expression, $\mathrm{NO}$, and inflammatory factor release, as well as NF- $\kappa \mathrm{B}$ translocation and caspase-related apoptosis in an ischemic/reperfusion neuron model [79]. Galla chinensis in TCM theory astringes, promotes wound healing and is used for ulcers and edema [24-26].

For Catechu, see above.

A gargle solution composed by these 5 herbs as well as saline gargle solution was administered in a randomised, controlled, two-armed clinical trial $(n=101$; treatment: $n=53$; control: $n=48$ ) to chemotherapy patients, compared to Borax solution gargling alone. All patients received basic treatment with antibiotics and vitamin supplements not further described. Improvement on mucositis symptoms was seen in $96.2 \%$ of the treatment group and $76.1 \%$ of the control group, judged by subjective clinical scales [52]. Significant conclusions on this result cannot be drawn as antibiotic regimens may have interfered with possible antiinflammatory effects of the herbal solution used at the same time.
5.8. Yu Nu Jian (Chinese), Jade Woman Decoction (English). This classical TCM formula containing Gypsum fibrosum, Rehmanniae viridae radix, Anemarrhenae rhizoma, Ophiopogonis radix, and Achyranthis bidentatae radix, is described in Collected Treatises of Zhang Jing Yue [80]. It is used for inflammatory diseases including oral ulcerations [39, 40]. Gypsum fibrosum is traditionally used for burns and ulcers $[24,25]$ as well as for fever [24-26], and it showed antipyretic activity demonstrated against LPS-induced pyrexia in rats, while calcined Gypsum and $\mathrm{CaSO}_{4}$ did not have this effect [81]. Rehmanniae viridae radix had been traditionally used for exanthema, abscesses, and sore throats [24-26]. Compounds of Rehmanniae viridae radix were found to inhibit NO production and iNOS, PGE-2, IL-6, and COX-2 expression in RAW 264.7 macrophages [82]. Anemarrhenae rhizoma had been traditionally used for dry coughs and infectious diseases [24-26]. Nyasol, a compound of Anemarrhenae rhizoma, was found to reduce NO and PGE-2 production as well as mRNAlevels of TNF- $\alpha$ and IL-1 $\beta$ in LPS-stimulated microglia cells. P38 mitogen-activated protein kinase (p38MAPK) was inactivated and LPS-induced I- $\kappa \mathrm{B} \alpha$ degradation was suppressed [83]. Achyranthis bidentatae radix calms bleeding from the mucosa in TCM theory and is used for mouth soreness, epistaxis, and hematemesis [24-26]. Achyranthis bidentatae polysaccharides derived from Achyranthis were found to positively modulate murine dendritic cell maturation by cell surface molecules CD86 and CD40 and major histocompatibility complex II (MHC II) enhancement and increase IL12 production, indicating a possible immune boosting effect [84].

As Yu Nu Jian was investigated with Qing Wei San (see below) in one study, oral mucositis related results are stated below.

5.9. Qing Wei San (Chinese), Clear the Stomach Powder (English). This classical TCM formula contains Coptidis rhizoma, Cimicifugae rhizoma, Rehmanniae viridae radix, Moutan cortex, Angelicae sinensis radix, and Achyranthis bidentatae radix. It is described in Lan Shi Mi Cang (Secrets from the Orchid Chamber) [74] and used for gingivitis and inflammations of tongue and lips.

In terms of TCM theory, Coptidis rhizoma is regularly used in inflammatory and septic processes [24-26]. It was found to inhibit IL-1 $\alpha$, IL-6, and granulocyte macrophage colony-stimulating factor (GM-CSF) secretion, iNOS expression, and NO production in RAW 264.7 macrophages [85]. Cimicifugae rhizoma in TCM terms removes toxicity and is used in exanthema, mucosal inflammation, and ulceration [24-26]. It was found to reduce LPS-induced release of IL6 , TNF- $\alpha$, IFN- $\gamma$, and stimulation of IL- 8 in LPS-induces human blood cells [86]. Moutan cortex in TCM terms is used in cases of inflammation and mucosal bleeding [24-26]. It was found to inhibit the activation of several inflammationrelated genes in gingival fibroblasts [87]. Angelica sinensis radix is used for sores, ulcers, and abscesses in TCM theory. Ligustilide, an Angelica sinensis radix compound, was found to suppress NO production, PGE-2, and TNF- $\alpha$ in LPSstimulated RAW 264.7 macrophages, to decrease activator 
protein-1 (AP-1), iNOS and NF- $\kappa$ B activation, phosphorylation of I $\kappa$ B kinase (IKK), MPAKs, ERK1/2, and c-Jun-Nterminale kinase (JNK) and to downwardly regulate intracellular reactive oxygen species (iROS) [88].

For Rehmanniae viridae radix and Achyranthis bidentatae radix, see above.

Both Yu Nu Jian (see above) and Qing Wei San were investigated in an uncontrolled case study on leukaemia patients $(n=31)$, clinically allocated into two groups according to the criteria of exuberance or deficiency by terms of TCM theory. $\mathrm{Yu} \mathrm{Nu}$ Jian was administered to the exuberance group while Qing Wei San was administered to the deficiency group. Additional topical medication was effected in all patients without further description. Ulcer grading was clinically judged. Reported without group differentiation, 7 patients obtained very good results and 19 patients offered good results on mucositis symptoms, indicating an improvement of $83.9 \%$ in summary [53]. In the context of a scientific study, the simultaneous use of different formulas precluded significant results. The findings require further investigation by higherquality trials.

5.10. Chrysanthemi flos Plus Gardeniae fructus Plus Hyperici perforati herba Plus Scrophulariae radix Plus Sophorae tonkinensis radix Combination. Classical combinations of these herbs have not been found. Obviously, the formula was empirically composed with the purpose of obtaining antiinflammatory and wound-healing effects. In terms of TCM theory, Gardeniae fructus had traditionally been externally used for wounds and contusions [24-26]. Geniposide from Gardeniae fructus were found to inhibit TNF- $\alpha$, IL-6, and IL$1 \beta$, to block the phosphorylation of $\mathrm{I} \kappa \mathrm{B} \alpha$ and transcription factor p65 and p38, as well as extracellular-signal-regulated kinases (ERK) and c-Jun N-terminal kinases (JNK), and to decrease the toll-like receptor-4 (TLR4) expression in LPSsimulated macrophages. They were also found to decrease the LPS-induced IL-8 production in human embryonic kidney cells (HEK293-mTLR4/MD-2 cells) [89]. Hyperici perforati herba is astringent and expels toxins in TCM theory [26]. Hyperici perforati herba compounds were found to inhibit LPS-induced PGE-2, COX-2, and NO through the suppression of cytokine signaling 3 (SOCS3) activation in 264.7 macrophages [90]. Scrophulariae radix in TCM theory is classified as removing toxicity and moistening tissues; it is used for sores and exanthema [24-26]. Sophorae tonkinensis radix had been traditionally used for tonsillitis, pharyngitis, and mouth sores $[26,91]$. It is toxic in higher doses. In vitro, it showed antiviral activity on Coxsackie-, Echo-, and Poliovirus [92].

For Chrysanthemi flos, see above.

As this prescription was investigated in one study with Qing Dai San (see below), mucositis related results are stated below.

5.11. Qing Dai San. Borax and Borneolum combination is found in Bing Peng San (Chinese), Borneol and Borax Powder (English), Pulvis Borneoli et Boracis (Latin), as a locally used prescription for oral inflammation and aphthous ulceration from Yi Zong Jin Jian (Golden Mirror of the Medical Tradition) [93]. An alternative to this prescription, Qing Dai San contains Borax, Borneolum, and Indigo naturalis [39, 40]. Borax is an extract of natural borax mineral. In terms of TCM theory, it removes toxicity from skin and mucosa upon external application [24, 25]. Borax compounds were found to reduce genotoxic effects of heavy metal exposure of human blood cell cultures by arsenic, bismuth, cadmium, mercury, and lead, normalising decreased antioxidant enzyme activities as well as sister chromatid exchange and micronuclei and plasma malondialdehyde (MDA) levels [94]. High Borax doses showed toxic cellular effects, decreasing human lymphocyte proliferation and increasing sister chromatid exchange in chromosomes [95].

Indigo naturalis is the fermented and chalked extract of Strobilenthes flaccidifolius, Indigo tinctoria, Isatis oblongata or Polygonaum tinctorium. It is used for exanthema and ulcers in TCM [24-26] and was found to to inhibit superoxide anione generation as well as MPAK phosphorylation and calcium mobilisation in formyl-methionyl-leucylphenylalanine (FMLP)-activated human neutrophils [96] .

\section{For Borneolum, see above.}

The compositions 5.10 and 5.11 were investigated together in a randomised, controlled, four-armed clinical trial on both chemotherapy- and radiation-induced oral mucositis. This article refers only to the two-armed part concerning chemotherapy patients $(n=60$; treatment: $n=30$; control: $n=30$ ). The decoction of Chrysanthemi flos, Gardeniae fructus, Hyperici perforate herba, Scrophulariae radix, and Sophorae tonkinensis radix was orally administered to the treatment group who also used a gargle solution composed of Borax, Borneolum, and Indigo naturalis. The control group patients received vitamin B12 administration and used a gentamycin/sodium//bicarbonate gargle solution. Ulcer grading was defined by size and quantity of ulcers. Improvement on oral mucositis symptoms were found in $96.7 \%$ of the herbal treatment group, compared to $86.7 \%$ in the control group. An overall shorter healing period for the herbal treatment group was reported [54]. In this trial, the investigation of two formulas at the same time compared to disparate controls impeded clear results.

5.12. Codonopsitis radix Plus Atractylodis macrocephalae Rhizome Plus Glycyrrhizae radix Plus Angelicae sinensis radix Plus Rehmanniae viridae radix Plus Astragali membranacei radix Plus Dioscoreae oppositae rhizoma Plus Alismatis rhizoma Plus Agastachis herba Plus Lophatheri herba. This formula may be regarded as a modified incomplete $\mathrm{Ba}$ Zhen Tang (Chinese), Eight Treasure Tea (English), Decoctum octo Gemmarum (Latin), from Zhen Ti Lei Yao (Catalogued Essentials for Correcting the Body) [97], which is a strong formula for devitalised patients, composed by Ginseng radix (or alternatively Codonopsitis radix), Atractylodis macrocephalae rhizoma, Glycyrrhizae radix, Angelicae sinensis radix, Rehmanniae radix, Poria alba, Paeoniae alba radix, and Ligustici chuangxiong rhizoma $[39,40]$. In prescription 5.12, the last three herbs of Ba Zhen Tang were replaced by Astragali membranacei radix, Dioscoreae oppositae radix, 
Alismatis rhizoma, Agastachis herba, and Lophatheri herba, directing the prescription to immune consolidating effects [24-26].

Agastachis herba, Atractylodis macrocephalae radix, and Glycyrrhizae radix are found in Huo Xiang Zheng Qi San (Chinese), Agastache Powder to Rectify the Qi (English), Pulvis Agastachis pro Qi Orthopathico (Latin) from Tai Ping Hui Min He Ji Ju Fang (Imperial Grace Formulary of the Tai Ping Era) [98] with Magnoliae cortex, Citri reticulatae pericarpium, Perillae folium, Angelica dahuricae radix, Pinelliae rhizoma, Arecae pericarpium, Poria alba, and Platycodonis radix. It is used for endemic infections and gastritis [39, 40]. Alismatis rhizoma, Codonopsitis radix, Atractylodis macrocephalae rhizoma, Angelicae sinensis radix, and Glycyrrhizae radix are also found in Dang Gui Nian Tong Tang (Chinese), Decoction to Lift the Pain (English) from Nei Wai Shang Bian Huo Lun (Clearing Doubts about Injury from Internal and External Causes) [99] with Atractylodis radix, Ledebouriellae radix, Puerariae radix, Scutellariae radix, Anemarrhenae rhizoma, Artemisiae herba, Polyporus, Sophorae radix, Notopterygii radix, and Cimicifugae radix. It is used for inflammatory diseases such as arthritis, impetigo, and eczema [100].

Codonopsitis radix extract was found to inhibit NO, TNF$\alpha$, IL-3 IL-6, and the ERK signalling pathway as well as LPS-induced phagocytic uptake and CD29-mediated cellcell-adhesion in RAW 264.7 macrophages [101]. Dioscoreae oppositae rhizoma was found to decrease the NO and proinflammatory cytokine production including IL- $1 \beta$, Il6 , TNF- $\alpha$, and PGE-2, as well as iNOS, and the COX-2, and NF- $\kappa \mathrm{B}$ activation in RAW 264.7 macrophages [102]. Alismatis rhizoma is diuretic in TCM theory [24-26]. It is not typically used for oral diseases but was found to suppress NF- $\kappa \mathrm{B}, \mathrm{COX}-2$, IL- $1 \beta$ and $\mathrm{iNOS}$, as well as induced nuclear factor-like 2 (Nrf2)-regulated gene expression in RAW 264.7 cells [103]. Agastachis herba in TCM theory has antiedematous effects and is used for nausea and fever [24, 25]. Agastachis herba extract showed antioxidant effects increasing heme oxygenase-1 (HO-1) enzyme activity by way of the protein kinase $\mathrm{G}$ (PKG) signalling pathway in RAW 264.7 macrophages [104]. Lophatheri herba has been traditionally used for mouth and tongue sores [26]. Glycosides derived from Lophatheri herba were found to possess an antirespiratory syncytial virus (RSV) effect in vitro [105].

For Atractylodis macrocephalae rhizoma, Glycyrrhizae radix, Astragali membranacei radix, Angelicae sinensis radix, and Rehmanniae viridae radix, see above.

The decoction of Codonopsitis radix, Atractylodis macrocephalae rhizoma, Glycyrrhizae radix, Angelicae sinensis radix, Rehmanniae viridae radix, Astragali membranacei radix, Dioscoreae oppositae rhizoma, Alismatis rhizoma, Agastachis herba, and Lophatheri herba was administered in a randomised, controlled, two-armed clinical trial $(n=66$; treatment: $n=33$; control: $n=33$ ) to chemotherapy patients during 4 chemotherapy cycles. The treatment group received not only the herbal decoction but also a multi vitamin, mineral, and micronutrient supplement (Centrum Wyeth). The control group patients received only vitamin B2 administration. Oral ulcer incidence grew from the first to the fourth chemotherapy cycle up to $21.2 \%$ in the treatment group and $66.7 \%$ in the control group, judged by WHO scale for Oral Mucositis. The increase was not only higher but also faster in the control group [55].

In this trial, the diversified vitamin supplement application confused the effect of the herbal medicinal treatment. Vitamin B2 is not a valuable control to any multivitamin supplement and/or Chinese medicinal herbs. Basic conditions should be equal in both groups in order to achieve a measurable effect. The effect of vitamin supplements should be investigated independently from herbal medicine.

5.13. Modified Xie Huang San (Chinese), Drain the Yellow Powder (English). The classical prescription Xie Huang San contains Gypsum fibrosum, Saposhnikoviae radix, Gardeniae fructus, Agastachis herba, and Glycyrrhizae radix. It is described in Xiao Er Yao Zheng Zhi Jue (Craft of Medicines and Patterns for Children) [106] and used for inflammatory diseases of stomach and mouth [39, 40].

Saposhnikoviae radix is used for affections of skin and mucosa [24-26]. It was found to inhibit NO production through iNOS and its mRNA expression in LPS-induced RAW 264.7 cells [107].

For the further herbs of this formula, see above.

Modifying standard prescriptions by herb addition related to syndrome patterns or individual symptoms is common in TCM tradition $[39,40]$. In a clinical trial on 90 chemotherapy patients [56], Xie Huang San was administered amended by addition of Ginseng radix, Astragali membranacei radix, Atractylodis macrocephalae rhizoma, Coptidis rhizoma, Taraxaci herba, Dendrobii caulis, Hedyotidis herba, and Lophatheri herba which offer additional anti-inflammatory qualities [24-26].

In terms of TCM theory, Taraxaci herba is used for swelling, abscesses, and sore throat [24-26]. Taraxacosterol a flavonoid, isolated from Taraxaci herba, was found to inhibit NO, PGE-2, TNF- $\alpha$, IL- $1 \beta$, and IL- 6 production as well as LPS-induced NF- $\kappa$ B translocation in RAW 264.7 macrophages [108]. Dendrobii caulis had been used for dry mouth in TCM theory [26]. Anti-inflammatory and saliva secretion increasing effects of Dendrobii caulis were indicated in a Sjögren's mouse model [109]. Hedyotis herba in TCM theory has been used for abscesses and ulcers [24, 25].

For Ginseng radix, Atractylodis macrocephalae rhizoma, Coptidis rhizoma, and Lophatheri herba, see above.

Astragali membranacei radix, Atractylodis macrocephalae rhizome, and Ginseng radix are found in Bu Zhong Yi Qi Tang (Chinese), Tonify the Middle and Augment the Qi Decoction (English), Decoctum Suppleens Centrum et Augmentans Qi (Latin) from Nei Wai Shang Bian Huo Lun (Clearing Doubts about Injury from Internal and External Causes) [99] with Glycyrrhizae radix tosta, Angelicae sinensis radix, Aurantii pericarpium, Cimicifugae rhizoma, and Bupleuri radix. Furthermore, Yu Ping Feng San (Chinese), Jade Windscreen Powder (English), Pulvis Paraventi Jaspidis (Latin) from Shi Yi De Xiao Fang (Effective Formulas from Generations of Physicians) [110] composed of Astragali membranacei radix, Atractylodis macrocephalae radix, and Saposhnikoviae radix, 
traditionally considered as consolidating the body's immune defence, is present in this multiple decoction.

This complex formula was investigated in the abovementioned randomised, controlled, two-armed clinical trial on chemotherapy patients ( $n=90$; treatment: $n=50$; control: $n=40$ ) by oral administration to the treatment group [56]. In addition, several of their individual symptoms were considered in each case by adding herbal modules. In case of diarrhoea, neutropenia, thrombopenia, petechiae, lymphadenopathy, fever, insomnia, night sweat, or increased ministerial fire (which is a specific term of TCM theory), two different herbs were added to the recipe.

This total of herbal medicine was compared to oral administration of vitamin B2 and vitamin C in the control group. All patients received dental care instructions and diet advices. Ulcer degree was classified by a subjective clinical scale.

Though positive effects on mucositis symptoms were seen in $98 \%$ of the treatment group compared to $72 \%$ of the vitamin control group, the formula complexity does not allow congruent conclusions due to several variances in the treatment group. The multiple interactions between single herbal components do not allow a clear view on the (formula's) effects. Furthermore, vitamin application may be useful for oral mucositis patients but does not represent a valid control to any complex herbal prescription of this size.

\section{Discussion}

Chemotherapy-induced oral mucositis continues to be a challenge for anticancer treatment $[8,9,12-15]$, representing one of the most common problems for chemotherapy patients [1]. Despite of the dedicated research on this field [1-7] and some resulting guidelines for prevention and treatment of oral mucositis $[9,10,16,17]$, therapeutic results are not yet satisfactory. Chemotherapy side effects in general represent an additional physical and psychological burden to patients diagnosed with cancer, reducing their quality of life and leading to the risk of anticancer treatment delay with fatal consequences $[7,8]$. On the other hand, posttraumatic stress reactions as seen in cancer patients lead to a decreased defence against oral mucositis, determined by the alteration of several biomarkers $[6,7]$.

Medicinal herbs are commonly used for complementary treatment when there is no sufficient western treatment concept. A Chinese review identified diverse approaches regarding oral mucositis by applying herbal medicine, including various gargling preparations, sprays, formulas for oral administration, acupoint application or intravenous injection, resulting in a generally positive effect [111], but the evidence of herbal use on oral mucositis still remains unclear.

Generally, systematic review studies on Chinese herbal medicine come to the conclusion that better qualified studies are necessary [112-114]. At the clinical level, study designs used to be mostly suboptimal but even on pharmacological level, the study quality is criticized as being sufficient. This is not surprising, because classical pharmacological research is generally focusing on single active compounds and this method of approach is not easily transferred to the multidimensional complexity of Asian herbal prescriptions. But in some aspects this view is short sighted, as a single-target approach can have limited effectiveness, and there is some evidence that a multi-target approach might be more effective $[115,116]$ and mixtures may have potentiating actions of their multiple bioactive components [117].

In this review, on the one hand we tried to summarize the state of knowledge of Chinese herbal treatment for chemotherapy-induced oral mucositis based on clinical trials. On the other hand, we tried to examine the TCM tradition based rationality of the particular herbs used for mucositis.

While aggressive treatments like chemotherapy have not been used in the history of TCM, application of traditional Chinese herbal treatment to these side effects of modern therapy requires an intentional transfer of historical concepts to modern treatment procedures. In daily practice, Chinese herbal medicine has an individualized approach that cannot be easily transferred into standardized controlled trials due to the uniform treatment concepts usually required by controlled trials.

For a full understanding of the mechanisms of herbal prescriptions, the effects of every single herb must be known on a molecular basis. Based on these data, herbal combinations should be investigated for detecting synergisms that may result in molecular effects which are not found in the single herb components $[118,119]$. Further elaborated research is necessary for clearing multiple questions about single as well as combined herbal use, resulting in the aim of rational prescription rather than application based only on empirical knowledge.

Even though all reviewed clinical trials reported positive effects of Chinese herbal treatment, they did not show adequate study designs proportionately with regard to the investigated questions. Some studies used complex multi-herbal formulas that lead to difficulties in understanding the detailed effects. Formula complexity should be well elaborated in order to achieve significant results. Monoherbal applications offer clear results on the basis of well elaborated study designs. Herbal combinations may be even more effective in clinical results [115-117], while under clinical study conditions the evidence of exaggerated multi-herbal application is narrowed by numerous interferences between the single compounds, especially in combined prescriptions of variant formulas (see $5.2,5.10,5.11,5.12$, and 5.13).

The use of individualized herbal combinations has a long tradition in TCM on an empirical basis. One of the evaluated trials tries to take this classical approach into account by additional prescriptions depending on the accompanying symptomatology, resulting in extremely complex treatment procedures (see 5.13). In consequence, the general overview gets lost. In other trials treatment procedures of topical application and internal or even intravenous administration were combined (see 5.1a, 5.3, 5.6, and 5.10 and 5.11), making it impossible to draw applicable conclusions about one of the interventions. One trial used a formula mixed with vitamins (see 5.12), while others used vitamin B2, B12, and/or vitamin $\mathrm{C}$ application for controls without discussing the rationale (see 5.3, 5.6, 5.11, 5.12, and 5.13). In the same manner, it is 
difficult to judge the efficacy on herbal ice application versus ambient tempered gargle solution (see 5.4). Antibiotics were used for control groups in some studies (see 4.3, 5.6, and 5.11) while basic use of antibiotics challenged the result of one study (see 5.7).

Only in one study a non-treatment group had been established (see 4.2) and in only 6 studies the same type of application was used for treatment as well as for control groups (see 4.3, 4.4, 5.1b, 5.2, 5.5, and 5.7). Two authors reported uncontrolled case studies (see 4.1, 5.8, and 5.9).

In summary it is almost impossible to evaluate which parts of the treatment concepts are responsible for the measured effects in the reviewed trials.

Another problem for judging study results is the fact that oral mucositis is a severe but short term side effect of chemotherapy, typically self-limiting in about 2 to 4 weeks if not complicated by infections [1]. Study designs have to consider that even without any specific treatments, symptoms possibly improve in this time.

In general, the investigated trials showed low-quality designs. Control groups were established in most investigated trials but control interventions did not represent any standards. It has to be admitted that the mucositis guidelines so far existing are limited, so it is not easy to find valid control interventions for some treatment concepts. But in general, clinical studies should offer a standard basic treatment for all patients or placebo non-treatment groups in order to gather valid data on the investigated intervention. In the case of mucositis, establishing non-treatment groups could cause an ethical dilemma for having possible severe consequences [9, 12,13 ] but it is possible to establish basic treatment conditions for all patients taking part of the study which do not interfere with the investigated intervention. This approach includes placebo administration for control groups in order to achieve valid data. In the case of Chinese medicinal herbs, the use of capsules containing herbal extractions or placebo is a good option. In the case of gargling solutions, fabricating a valid placebo may be more complicated but should not be impossible. Patients and medical practitioners should be blinded regarding the applied intervention in order to minimize placebo effects. The blinding technique has not been reported in any of the investigated studies. Randomisation has been reported in all controlled studies though the process has not been described except very briefly in two publications (5.1b and 5.12). For proving a strict randomisation protocol so as not to create bias, it is necessary to provide a detailed description.

Summarizing the collected data so far, results of Chinese medicinal herbal administration for chemotherapyinduced oral mucositis are potentially promising, but poor study designs do not allow valid conclusions. Conducting a meta-analysis is not possible with the present database. Further investigations are necessary on molecular mechanisms of multi-herbal formulas and the corresponding single herbs as well as in well designed clinical trials. Providing adequate study designs are developed, traditional Chinese herbal medicine has the potential of complementing western regimens such as chemotherapy in order to achieve lower levels of side effects, thus enabling patients to better resist chemotherapy impacts.

\section{Conclusion}

All evaluated trials in this review reported positive effects about Chinese herbal treatment for chemotherapy-induced oral mucositis, but the value of these treatments remains unclear. Study designs are generally poor, some herbal prescriptions are far too complex and adequate controls are missing. Mechanisms of action are rarely described.

While basic research provides data about antiinflammatory and protective effects of some herbs or herbal compounds, further research is still promising, but study designs need considerable improvement. So future research should start with mechanism based studies first. The following clinical studies should reduce the complexity of the treatment procedures in order to produce clear results, before Chinese herbal medicine can become an evidenced based part of the treatment of chemotherapy induced mucositis.

\section{Conflict of Interests}

The authors declare that they have no conflict of interests.

\section{References}

[1] National Cancer Institute, http://www.cancer.gov/cancertopics/ pdq.

[2] M. Rostock and R. Saller, "Schleimhautschäden unter antitumoraler Behandlung: Präventive und therapeutische Möglichkeiten mit pflanzlichen Zubereitungen," Schweizerische Zeitschrift für Ganzheitsmedizin, vol. 19, no. 4, pp. 212-217, 2007.

[3] J. B. Epstein, A. H. F. Tsang, D. Warkentin, and J. A. Ship, "The role of salivary function in modulating chemotherapyinduced oropharyngeal mucositis: a review of the literature," Oral Surgery, Oral Medicine, Oral Pathology, Oral Radiology, and Endodontics, vol. 94, no. 1, pp. 39-44, 2002.

[4] C. Scully, J. Epstein, and S. Sonis, "Oral mucositis: a challenging complication of radiotherapy, chemotherapy, and radiochemotherapy. Part 1, pathogenesis and prophylaxis of mucositis," Head and Neck, vol. 25, no. 12, pp. 1057-1070, 2003.

[5] D. E. Peterson and R. V. Lalla, "Oral mucositis: the new paradigms," Current Opinion in Oncology, vol. 22, no. 4, pp. 318322, 2010.

[6] C. Xu, J. Zhao, W. T. Loo et al., "Correlation of epigenetic change and identification of risk factors for oral submucous fibrosis," International Journal of Biological Markers, vol. 27, no. 4, pp. e314-e321, 2010.

[7] L. J. Bai, Q. Liu, M. Wang et al., "Evaluation of the psychological and biological changes of patients diagnosed with benign and malignant breasttumors," International Journal of Biological Markers, vol. 27, no. 4, pp. e322-e330, 2012.

[8] D. McGowan, "Chemotherapy-induced oral dysfunction: a literature review," British Journal of Nursing, vol. 17, no. 22, pp. 1422-1426, 2008.

[9] C. Wojtaszek, "Management of chemotherapy-induced stomatitis," Clinical Journal of Oncology Nursing, vol. 4, no. 6, pp. 263270, 2000. 
[10] D. M. Keefe, M. M. Schubert, L. S. Elting et al., "Updated clinical practice guidelines for the prevention and treatment of mucositis," Cancer, vol. 109, no. 5, pp. 820-831, 2007.

[11] Mucositis Study Section of the Multinational Association of Supportive Care in Cancer and The International Society for Oral Oncology, "Mucositis, perspectives and clinical practice guidelines, perspectives on cancer therapx-induced mucosal injury," http://www3.interscience.wiley.com/cgi-bin/ fulltext/108069519.

[12] F. K. L. Spijkervet and S. T. Sonis, "New frontiers in the management of chemotherapy-induced mucositis," Current Opinion in Oncology, vol. 10, supplement 1, pp. S23-S27, 1998.

[13] S. A. Khan and J. R. Wingard, "Infection and mucosal injury in cancer treatment," Journal of the National Cancer Institute. Monographs, no. 29, pp. 31-36, 2001.

[14] WHOHandbook, 1979.

[15] S. T. Sonis, L. S. Elting, D. Keefe et al., "Perspectives on cancer therapy-induced mucosal injury," Cancer, vol. 100, supplement, no. 9, pp. 1995-2025, 2004.

[16] R. V. Lalla, S. T. Sonis, and D. E. Peterson, "Management of oral mucositis in patients with cancer," Dental Clinics of North America, vol. 52, no. 1, pp. 61-77, 2008.

[17] 2013, http://www.mascc.org/mucositis-guidelines.

[18] H. V. Worthington, J. E. Clarkson, G. Bryan et al., "Interventions for preventing oral mucositis for patients with cancer receiving treatment," Cochrane Database of Systematic Reviews, vol. 12, p. CD000978, 2010.

[19] H. V. Worthington, J. E. Clarkson, G. Bryan et al., "Interventions for preventing oral mucositis for patients with cancer receiving treatment," Cochrane Database of Systematic Reviews, vol. 4, p. CD000978, 2011.

[20] E. Ernst and B. R. Cassileth, "The prevalence of complementary/alternative medicine in cancer: a systematic review," Cancer, vol. 83, no. 4, pp. 777-782, 1998.

[21] H. Boon, J. B. Brown, A. Gavin, M. A. Kennard, and M. Stewart, "Breast cancer survivors' perceptions of complementary/alternative medicine (CAM): making the decision to use or not to use," Qualitative Health Research, vol. 9, no. 5, pp. 639653, 1999.

[22] H. A. Tindle, R. B. Davis, R. S. Phillips, and D. M. Eisenberg, "Trends in use of complementary and alternative medicine by us adults: 1997-2002," Alternative Therapies in Health and Medicine, vol. 11, no. 1, pp. 42-49, 2005.

[23] C. W. H. Chan, A. M. Chang, A. Molassiotis, I. Y. M. Lee, and G. C. T. Lee, "Oral complications in Chinese cancer patients undergoing chemotherapy," Supportive Care in Cancer, vol. 11, no. 1, pp. 48-55, 2003.

[24] M. Porkert, Klinische Chinesische Pharmakologie, Phainon, Dinkelscherben, Germany, 2nd edition, 1994.

[25] D. Bensky, S. Clavey, and E. Stoger, Chinese Herbal Medicine: Materia Medica, Eastland Press, Seattle, Wash, USA, 3rd edition, 2004.

[26] Chinese Pharmacopoeia Commision, Pharmakocoeia of the People' Republic of China, China Medical Science Press, Beijing, China, 2010.

[27] H. Yu, H. Jin, W. Gong, Z. Wang, and H. Liang, "Pharmacological actions of multi-target-directed evodiamine," Molecules, vol. 18, no. 2, pp. 1826-1843, 2013.

[28] T. L. Xu and J. H. Han, "External application of Evodia rutaecarpa on Acupoint Yongquan in remaining dental ulcer after chemotherapy. Zhu Yu waifu yong quam xue ke zhiliao hualiao hou kouqiang kui yang," Chinese Journal of Nursing, vol. 41, no. 10, p. 837, 2006.

[29] http://www.shen-nong.com/eng/herbal/hongjingtian.html .

[30] H. X. Li, S. C. W. Sze, Y. Tong, and T. B. Ng, "Production of Th1and Th2-dependent cytokines induced by the Chinese medicine herb, Rhodiola algida, on human peripheral blood monocytes," Journal of Ethnopharmacology, vol. 123, no. 2, pp. 257-266, 2009.

[31] W. T. Y. Loo, L. Jin, L. W. C. Chow, M. N. B. Cheung, and M. Wang, "Rhodiola algida improves chemotherapy-induced oral mucositis in breast cancer patients," Expert Opinion on Investigational Drugs, vol. 19, supplement 1, pp. S91-S100, 2010.

[32] http://www.naturalremedy.com/Acacia\%20catechu.pdf.

[33] J. Tseng-Crank, S. Sung, Q. Jia et al., "A medicinal plant extract of scutellaria baicalensis and acacia catechu reduced LPSstimulated gene expression in immune cells: a comprehensive genomic study using QPCR, ELISA, and microarray," Journal of Dietary Supplements, vol. 7, no. 3, pp. 253-272, 2010.

[34] Y. J. Shi and J. Z. Shan, "Observation on the effect of catechin from traditional Chinese medicine on oral ulcer induced by chemotherapy," Journal of Clinical Nursing, vol. 8, no. 1, p. 47, 2009.

[35] H. X. Chen, X. H. Liang, and L. H. Jiang, "Clinical effect of Kangfuxin liquid in treatment of recurrent aphtous ulcer," China Practical Medicine, no. 3, 2011.

[36] L. M. Wang, Y. M. Lu, J. Yu, D. S. Zhu, and W. X. Chen, "Inhibition of the expression of cytokines on experimental colitis in mice by kangfuxin," Chinese Journal of Clinical Pharmacology, no. 3, 2006.

[37] H. Wang, X. F. Xu, Z. W. Zeng et al., "Rehabilitation drug solution on prevention and treatment of chemotherapy-induced oral ulcers," Medical Information, no. 12, p. 3742, 2010.

[38] J. T. Wu, Wen Bing Tiao Bian (Systematic Differentiation of Warm Diseases), vol. 1798, Nanjing University of TCM, Warm Diseases Department; People's Medical Publishing House, 2005.

[39] V. Scheid, D. Bensky, A. Ellis, and R. Barolet, Chinese Herbal Medicine: Formulas and Strategies, Eastland Press, Seattle, Wash, USA, 2nd edition, 2004.

[40] M. Porkert, Klassische Chinesische Rezeptur, Phainon, Dinkelscherben, Germany, 2nd edition, 1994.

[41] Y. Liao, S. Dong, R. Kijama, P. Cai, L. Liu, and H. Shen, "Flos lonicerae exracts and chlorogenic acid protect human umbilical vein endothelia cells from the toxic damage of perfluorooctane sulphonate," Inflammation, vol. 36, no. 3, pp. 767-779, 2013.

[42] E. M. Shin, H. Y. Zhou, L. Y. Guo et al., "Anti-inflammatory effects of glycyrol isolated from Glycyrrhiza uralensis in LPSstimulated RAW264.7 macrophages," International Immunopharmacology, vol. 8, no. 11, pp. 1524-1532, 2008.

[43] Y. W. Kim, R. J. Zhao, S. J. Park et al., "Anti-inflammatory effects of liquiritigenin as a consequence of the inhibition of NF- $\kappa$ Bdependent iNOS and proinflammatory cytokines production," British Journal of Pharmacology, vol. 154, no. 1, pp. 165-173, 2008.

[44] Z. Q. Ma and X. H. Song, "Licorice and Honeysuckle decoction for prevention oral ulcer by high dose chemotherapy: a clinical observation. Gan cao Jin Hua jian yin yufang da ji liang hualiao kouqiang kuiyang de linchuang guancha," Modern Journal of Lntegrated Traditional Chinese and Western Medicine, no. 18, pp. 2408-2409, 2005.

[45] Y. X. Zeng, "Yin Hua Gan Cao decoction was used to treat stomatitis caused by chemical therapy. Yin Hua Gan Cao Tang yongyu hualiao bingren kouqiang yan de huli tihui," Guiding Journal of TCM, vol. 11, no. 12, pp. 50-51, 2005. 
[46] A. Nalbantsoy, T. Nesil, O. Yilmaz-Dilsiz, G. Aksu, S. Khan, and E. Bedir, "Evaluation of the immunomodulatory properties in mice and in vitro anti-inflammatory activity of cycloartane type saponins from Astragalus species," Journal of Ethnopharmacology, vol. 139, no. 2, pp. 574-581, 2012.

[47] N. X. Bao, G. Z. Zeng, L. W. Zhou, X. Y. Shu, and L. Yang, "Preventive effect of self-made gargle solution on chemotherapyinduced oral ulcer in patients with colorectal carcinoma," Journal of Nursing Science, vol. 23, no. 20, pp. 44-45, 2008.

[48] J. Y. Chen, A. L. Peng, G. Z. Hao, H. Zhu, and Y. Z. Yao, "Curative effect of ophiopogonon tuber mixture on ulcer of oral cavity after chemotherapy. Mai Dong He Ji zhiliao hualiao hou kouqian kuiyang xiaoguo guancha," Journal of Nursing Science, vol. 20, no. 20, pp. 76-77, 2005.

[49] X. E. Wu Zhu YF, Y. M. Lai, and X. H. Xie, "Gargle medicine ice observation on the effects of chemotherapy in patients with oral ulcer," Journal of Nursing, vol. 16, no. 9, pp. 64-65, 2009.

[50] L. H. Wang, A. W. Dai, and L. Wang, "The effect of Shengmai injection on alleviating acute chemotherapy toxicity. Wu Zhu Yu waifu yongquan xue ke zhiliao hualiao hou kouqian kuiyang," New Journal of Traditional Chinese Medicine, vol. 38, no. 12, pp. 38-39, 2006.

[51] T. Jin, M. H. Shen, Y. F. Sun, and J. Zhang, "Water Grass Decoction for treating Chemotherapy-induced Oral Ulcers. Shui Zhong Cao Tang Ji zhiliao hualiao suozhi kouqiang kuiyang," Chinese Arcieves of Raditional Chinese Medicine, vol. 27, no. 2, pp. 303-305, 2009.

[52] F. J. Hou, B. C. Jin, and W. Li, "Observation on the effects of yellow five gargle for oral ulcers caused by chemotherapy. Huang Wu shukouye yongyu hualiao suoshi kouqiang kuiyang de xiaoguo guancha," Journal of Nursing Science, vol. 16, no. 8, pp. 494-495, 2001.

[53] H. Zhu and J. Zhang, "Treatment of stomatological complications in 31 cases of acute leukemia with Chinese herbal drugs," Journal of Traditional Chinese Medicine, vol. 13, no. 4, pp. 253256, 1993.

[54] Z. X. Zhou, Z. R. Zhang, H. X. Qian, Z. Q. Li, W. M. Wu, and J. Luo, "Traditional Chinese medicine in preventing and treating oral ulcer induced by chemotherapy clinical observation," Journal of Sichuan of Traditional Chinese Medicine, vol. 23, no. 10, pp. 93-94, 2005.

[55] C. Y. Chen and Y. Z. Zheng, "Clinical study on prevention of oral ulcers caused by chemotherapy with the combined therapy of centrum and specific Chinese herbs that invigorate the function of the spleen and regulate the flow of Qi," Chinese Journal of Clinical Nutrition, vol. 13, no. 6, pp. 398-400, 2005.

[56] J. H. Sun, "Clinical Observation of modified Xiehuang Powder in the Treatment of Oral Ulcer following Chemotherapy. Xie Huang San jiawei zhiliao hualiao hou kouqiang kuiyang linchuang guancha," Modern Journal of Integrated Traditional Chinese and Western Medicine, vol. 16, no. 7, pp. 900-901, 2007.

[57] A. Wang, Yi Fang Yi Jie (Analytic Collection of Medical Formulas), People's Medical Publishing House, 2006.

[58] X. Wu, H. Dai, L. Huang, X. Gao, K. W. K. Tsim, and P. Tu, “A fructan, from radix ophiopogonis, stimulates the proliferation of cultured lymphocytes: structural and functional analyses," Journal of Natural Products, vol. 69, no. 9, pp. 1257-1260, 2006.

[59] J. Y. Kim, Y. P. Hwang, D. H. Kim et al., "Inhibitory effect of the saponins derived from roots of Platycodon grandiflorum on carrageenan-induced inflammation," Bioscience, Biotechnology and Biochemistry, vol. 70, no. 4, pp. 858-864, 2006.
[60] M. S. Cheon, T. Yoon, D. Y. Lee et al., "Chrysanthemum indicum Linné extract inhibits the inflammatory response by suppressing NF- $\kappa$ B and MAPKs activation in lipopolysaccharideinduced RAW 264.7 macrophages," Journal of Ethnopharmacology, vol. 122, no. 3, pp. 473-477, 2009.

[61] L.-X. Sun, L.-H. Chen, Z.-B. Lin et al., "Effects of Ganoderma lucidum polysaccharides on IEC-6 cell proliferation, migration and morphology of differentiation benefiting intestinal epithelium healing in vitro," Journal of Pharmacy and Pharmacology, vol. 63, no. 12, pp. 1595-1603, 2011.

[62] L. Tie, H.-Q. Yang, Y. An et al., "Ganoderma lucidum polysaccharide accelerates refractory wound healing by inhibition of mitochondrial oxidative stress in type 1 diabetes," Cellular Physiology and Biochemistry, vol. 29, no. 3-4, pp. 583-594, 2012.

[63] H. M. Yoon, K. J. Jang, M. S. Han et al., "Ganoderma lucidum ethanol extract inhibits the inflammatory response by suppressing the NF-kB and toll-like receptor pathways in lipopolysaccharide-stimulated BV2 microglial cells," Experimental and Therapeutic Medicine, vol. 5, no. 3, pp. 957-936, 2013.

[64] R. W. K. Wong, U. Hägg, L. Samaranayake, M. K. Z. Yuen, C. J. Seneviratne, and R. Kao, "Antimicrobial activity of Chinese medicine herbs against common bacteria in oral biofilm. A pilot study," International Journal of Oral and Maxillofacial Surgery, vol. 39, no. 6, pp. 599-605, 2010.

[65] B. C. L. Chan, K. L. E. Hon, P. C. Leung et al., “Traditional Chinese medicine for atopic eczema: penta herbs formula suppresses inflammatory mediators release from mast cells," Journal of Ethnopharmacology, vol. 120, no. 1, pp. 85-91, 2008.

[66] C. A. Migliorati, L. Oberle-Edwards, and M. Schubert, "The role of alternative and natural agents, cryotherapy, and/or laser for management of alimentary mucositis," Supportive Care in Cancer, vol. 14, no. 6, pp. 533-540, 2006.

[67] Y. S. Zhang, Yu Xue Qi Yuan (Expounding on the Origins of Medicine): Yuan Dynasty, People's Military Medical Press, 2002.

[68] D. C. W. Lee and A. S. Y. Lau, "Effects of Panax ginseng on tumor necrosis factor- $\alpha$-mediated inflammation: a minireview," Molecules, vol. 16, no. 4, pp. 2802-2816, 2011.

[69] W. K. Kim, S. Y. Song, W. K. Oh et al., "Wound-healing effect of ginsenoside $\mathrm{Rd}$ from leaves of Panax ginseng via cyclic AMP-dependent protein kinase pathway," European Journal of Pharmacology, vol. 702, no. 1-3, pp. 285-293, 2013.

[70] H. Nishida, N. Tatewaki, Y. Nakajima et al., "Inhibition of ATR protein kinase activity by schisandrin B in DNA damage response," Nucleic Acids Research, vol. 37, no. 17, pp. 5678-5689, 2009.

[71] Y. Takimoto, H. Y. Qian, E. Yoshigai, T. Okomura, Y. Ikeya, and M. Nishizawa, "Gomisin N in the herbal drug gomishi (Schisandra chinensis) suppresses inductible nitric oxide synthase gene via C/EBPbeta and NF-kB in rat hepatocytes," Nitric Oxide, vol. 28, pp. 47-56, 2013.

[72] R. Liu, M. Wang, and J.-A. Duan, "Antipyretic and antioxidant activities of the aqueous extract of Cornu Bubali (water buffalo horn)," The American Journal of Chinese Medicine, vol. 38, no. 2, pp. 293-306, 2010.

[73] Y. Tu, L. Sun, M. Guo, and W. Chen, "The medicinal uses of Callicarpa L. in traditional Chinese medicine: an Ethnopharmacological, phytochemical and pharmacological review," Journal of Ethnopharmacology, vol. 146, no. 2, pp. 465-481, 2013.

[74] D. Y. Li, Lan Shi Mi Cang (Secrets From the Orchid Chamber), vol. 1336, People's Medical Publishing House, 2005.

[75] Y.-K. Park, Y. S. Chung, Y. S. Kim, O.-Y. Kwon, and T. H. Joh, "Inhibition of gene expression and production of iNOS 
and TNF- $\alpha$ in LPS-stimulated microglia by methanol extract of Phellodendri cortex," International Immunopharmacology, vol. 7, no. 7, pp. 955-962, 2007.

[76] K. Kim, S. Lee, S. Shin et al., "Anti-inflammatory function of arctiin by inhibiting COX-2 expression via NF- $\kappa$ B pathways," Journal of Inflammation, vol. 8, article 16, 2011.

[77] http://www.epharmacognosy.com/2012/08/european-verbenaherb-ma-bian-cao.html.

[78] E. Speroni, R. Cervellati, S. Costa et al., "Effects of differential extraction of Verbena officinalis on rat models of inflammation, cicatrization and gastric damage," Planta Medica, vol. 73, no. 3, pp. 227-235, 2007.

[79] R. Liu, L. Zhang, X. Lan et al., "Protection by borneol on cortical neurons against oxygen-glucose deprivation/reperfusion: involvement of anti-oxidation and anti-inflammation through nuclear transcription factor $\kappa$ appaB signaling pathway," Neuroscience, vol. 176, pp. 408-419, 2011.

[80] J. B. Zhang, Collected Treatise of [Zhang] Jing-Yue: 1624. Jing Yue Quan Shu, People's Medical Publishing House, 1997.

[81] H. D. Wang, M. Y. Wang, and X. B. Li, "Evaluation of the antipyretic activity of Gypsum Fibrosum and its constituents," Asian Journal of Traditional Medicines, vol. 4, no. 2, 2009.

[82] C. L. Liu, L. Cheng Ko CH, C. W. Wong et al., "Bioassayguided isolation of anti-inflammatory components from the root of Rehmannia glutinosa and ist underlying mechanism via inhibition of iNOS pathway," Journal of Ethnopharmacology, vol. 143, no. 3, pp. 867-875, 2012.

[83] H. J. Lee, H. Li, H. R. Chang, H. Jung, D. Y. Lee, and J. H. Ryu, "(-)-Nyasol, isolated from Anemarrhena asphodeloides suppresses neuroinflammatory response through the inhibition of I-alphaBa degradation in LPS-stimulated BV-2 microglial cells," Journal of Enzyme Inhibition and Medicinal Chemistry. In press.

[84] Y. Zou, J. Meng, W. Chen et al., "Modulation of phenotypic and functional maturation of murine dendritic cells (DCs) by purified Achyranthes bidentata polysaccharide (ABP)," International Immunopharmacology, vol. 11, no. 8, pp. 1103-1108, 2011.

[85] J. M. Kim, H. A. Jung, J. S. Choi, and N. G. Lee, "Identification of anti-inflammatory target genes of Rhizoma coptidis extract in lipopolysaccharide-stimulated RAW264.7 murine macrophage-like cells," Journal of Ethnopharmacology, vol. 130, no. 2, pp. 354-362, 2010.

[86] D. Schmid, F. Woehs, M. Svoboda, T. Thalhammer, P. Chiba, and T. Moeslinger, "Aqueous extracts of Cimicifuga racemosa and phenolcarboxylic constituents inhibit production of proinflammatory cytokines in LPS-stimulated human whole blood," Canadian Journal of Physiology and Pharmacology, vol. 87, no. 11, pp. 963-972, 2009.

[87] C. S. Yun, Y. G. Choi, M. Y. Jeong, J. H. Lee, and S. Lim, "Moutan cortex radicis inhibits inflammatory changes og gene expression in lipopolysaccharide-stimulated gingival fibroblasts," Journal of Natural Medicines, vol. 67, no. 3, pp. 576-589, 2012.

[88] H. J. Lee, H. Li, H. R. Chang, H. Jung, D. Y. Lee, and J. H. Ryu, "(-)-Nyasol, 10 isolated from Anemarrhena asphodeloides suppresses neuroinflammatory response through the inhibition of I-alphaBa degradation in LPS-stimulated BV-2 microglial cells," Journal of Enzyme Inhibition and Medicinal Chemistry, vol. 28, no. 5, pp. 954-959, 2013.

[89] Y. Fu, B. Liu, J. Liu et al., "Geniposide, from Gardenia jasminoides Ellis, inhibits the inflammatory response in the primary mouse macrophages and mouse models," International Immunopharmacology, vol. 14, no. 4, pp. 792-798, 2012.
[90] N. Huang, L. Rizshsky, C. C. Hauck, B. J. Nikolau, P. A. Murphy, and D. F. Birt, "The inhibition of lipopolysaccharide-induced macrophage inflammation by 4 compounds in Hypericum perforatum extract is partially dependent on the activation of SOCS3," Phytochemistry, vol. 76, pp. 106-116, 2012.

[91] C. H. Hempen and T. Fischer, Leitfaden Chinesische Phytotherapie, Elsevier, Amsterdam, Netherlands, 2nd edition, 2006.

[92] J.-P. Guo, J. Pang, X.-W. Wang, Z.-Q. Shen, M. Jin, and J.-W. $\mathrm{Li}$, "In vitro screening of traditionally used medicinal plants in China against enteroviruses," World Journal of Gastroenterology, vol. 12, no. 25, pp. 4078-4081, 2006.

[93] Q. Wu, Yi Zong Jin Jian (Golden Mirror of the Medical Tradition), vol. 1742, People's Medical Publishing House, 2006.

[94] H. Turkez, F. Geyikoglu, A. Tatar, M. S. Keles, and I. Kaplan, "The effects of some boron compounds against heavy metal toxicity in human blood," Experimental and Toxicologic Pathology, vol. 64, no. 1-2, pp. 93-101, 2012.

[95] M. Pongsavee, "Effect of borax on immune cell proliferation and sister chromatid exchange in human chromosomes," Journal of Occupational Medicine and Toxicology, vol. 4, no. 1, article 27, 2009.

[96] Y.-K. Lin, Y.-L. Leu, T.-H. Huang et al., "Anti-inflammatory effects of the extract of indigo naturalis in human neutrophils," Journal of Ethnopharmacology, vol. 125, no. 1, pp. 51-58, 2009.

[97] J. Xue, Zheng Ti Lei Yao (Catalogued Essentials For Correcting the Body), vol. 1529, Hainan International Press of World News, 1995.

[98] Tai Ping Hui Min He Ji Ju, Tai Ping Hui Min He Ji Ju Fang (Imperial Grace Formulary of the Tai Ping Era), 1107, People's Medical Publishing House, 2007.

[99] D. Y. Li, Nei Wai Shang Bian Huo Lun (Clearing Doubts About Injury From Internal and External Causes), vol. 1247, People's Medical Publishing House, 2007.

[100] http://www.tcmassistant.com.

[101] Y. G. Lee, J. Y. Kim, J. Y. Lee et al., "Regulatory effects of Codonopsis lanceolata on macrophage-mediated immune responses," Journal of Ethnopharmacology, vol. 112, no. 1, pp. 180-188, 2007.

[102] S. Kim, S. Shin, B. Hyun et al., "Immunmodulatory effects of dioscoreae rhizome against inflammation through suppressed production of cytokines via inhibition of the NF-kappaB pathway," Immune Network, vol. 12, no. 5, pp. 181-188, 2012.

[103] C. W. Han, M. J. Kwun, K. H. Kim et al., "Ethanol extract of alismatis rhizoma reduces acute lung inflammation by suppressing NF-kappaB and activating Nrf2," Journal of Ethnopharmacology, vol. 146, no. 1, pp. 402-410, 2013.

[104] H. M. Oh, Y. J. Kang, Y. S. Lee et al., "Protein kinase Gdependent heme oxygenase-1 induction by Agastache rugosa leaf extract protects RAW264.7 cells from hydrogen peroxideinduced injury," Journal of Ethnopharmacology, vol. 103, no. 2, pp. 229-235, 2006.

[105] Y. Wang, M. Chen, J. Zhang et al., "Flavone C-glycosides from the leaves of Lophatherum gracile and their in vitro antiviral activity," Planta Medica, vol. 78, no. 1, pp. 46-51, 2012.

[106] Y. Qian, Xiao Er Yao Zheng Zhi Jue (Craft of Medicines and Patterns For Children), vol. 1119, People's Publishing Medical House, 2007.

[107] J. Tai and S. Cheung, "Anti-proliferative and antioxidant activities of Saposhnikovia divaricata," Oncology Reports, vol. 18, no. 1, pp. 227-234, 2007. 
[108] X. Zhang, H. Xiong, and L. Liu, "Effects of taraxasterol on inflammatory responses in lipopolysaccharide- induced RAW 264.7 macrophages," Journal of Ethnopharmacology, vol. 141, no. 1, pp. 206-211, 2012.

[109] X. Lin, P.-C. Shaw, S. C.-W. Sze, Y. Tong, and Y. Zhang, "Dendrobium officinale polysaccharides ameliorate the abnormality of aquaporin 5, pro-inflammatory cytokines and inhibit apoptosis in the experimental Sjögren's syndrome mice," International Immunopharmacology, vol. 11, no. 12, pp. 2025-2032, 2011.

[110] Y. L. Wei, Shi Yi De Xiao Fang (Effective Formulas From Generations of Physicians), vol. 1345, Peoples Medical Publishing House, 2006.

[111] C. L. Wang, "Present situation of study on TCM prevention and treatment of oral ulcer induced by chemotherapy," Journal of Nursing, vol. 18, no. 3, pp. 16-19, 2011.

[112] J. P. Liu, M. Zhang, W. Y. Wang, and S. Grimsgaard, "Chinese herbal medicines for type 2 diabetes mellitus," Cochrane Database of Systematic Reviews, no. 3, p. CD003642, 2004.

[113] W. C. S. Cho, "Scientific evidence on the supportive cancer care with chinese medicine," Chinese Journal of Lung Cancer, vol. 13, no. 3, pp. 190-194, 2010.

[114] S. G. Li, H. Y. Chen, C. S. Ou-Yang et al., "The efficacy of Chinese herbal medicine as an adjunctive therapy, for advanced nonsmall cell lung cancer: a systematic review and meta-analysis," PLoS ONE, vol. 8, no. 2, Article ID e57604, 2013.

[115] X.-Y. Tian and L. Liu, "Drug discovery enters a new era with multi-target intervention strategy," Chinese Journal of Integrative Medicine, vol. 18, no. 7, pp. 539-542, 2012.

[116] J.- J. Lu, W. Pan, Y.-J. Hu, and Y.-T. Wang, "Multi-target drugs: the trend of drug research and development," PLOS ONE, vol. 7, no. 6, Article ID e40262, 2012.

[117] B. M. Schmidt, D. M. Ribnicky, P. E. Lipsky, and I. Raskin, "Revisiting the ancient concept of botanical therapeutics," Nature Chemical Biology, vol. 3, no. 7, pp. 360-366, 2007.

[118] L. Wu, X. P. Ding, D. N. Zhu, B. Y. Yu, and Y. Q. Yan, "Study on the radical scavengers in the traditional Chinese medicine formula Shengmai San by HPLC-DAD coupled with chemiluminescence (CL) and ESI-MS/MS," Journal of Pharmaceutical and Biomedical Analysis, vol. 52, no. 4, pp. 438-445, 2010.

[119] Y. Suzuki, K. Goto, A. Ishige, Y. Komatsu, and J. Kamei, "Antinociceptive effect of Gosha-jinki-gan, a Kampo medicine, in streptozotocin-induced diabetic mice," Japanese Journal of Pharmacology, vol. 79, no. 2, pp. 169-175, 1999. 


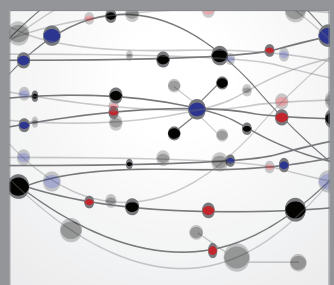

The Scientific World Journal
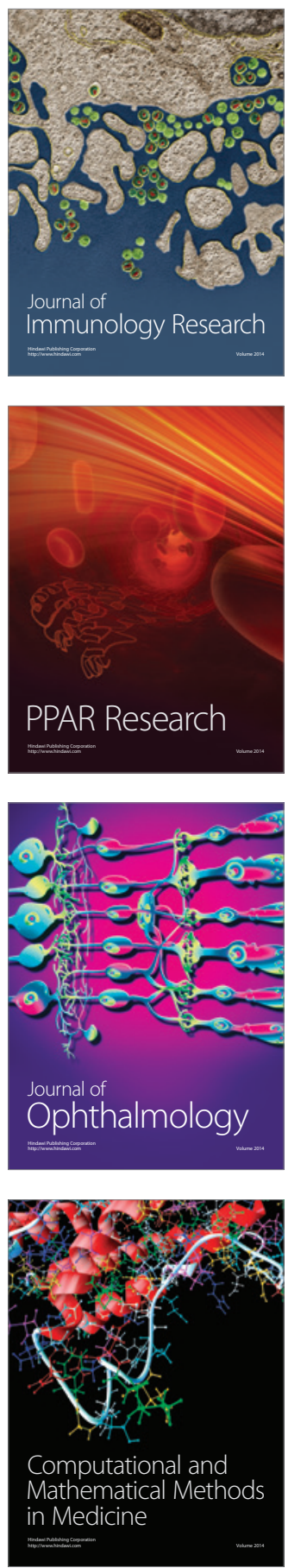

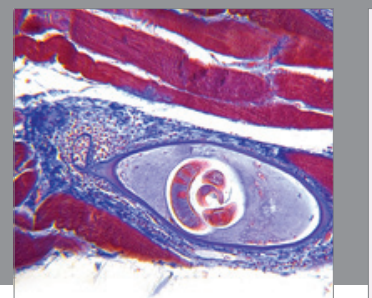

Gastroenterology

Research and Practice
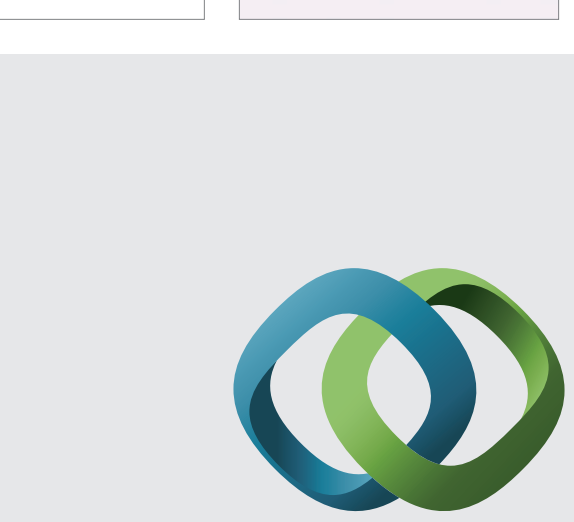

\section{Hindawi}

Submit your manuscripts at

http://www.hindawi.com
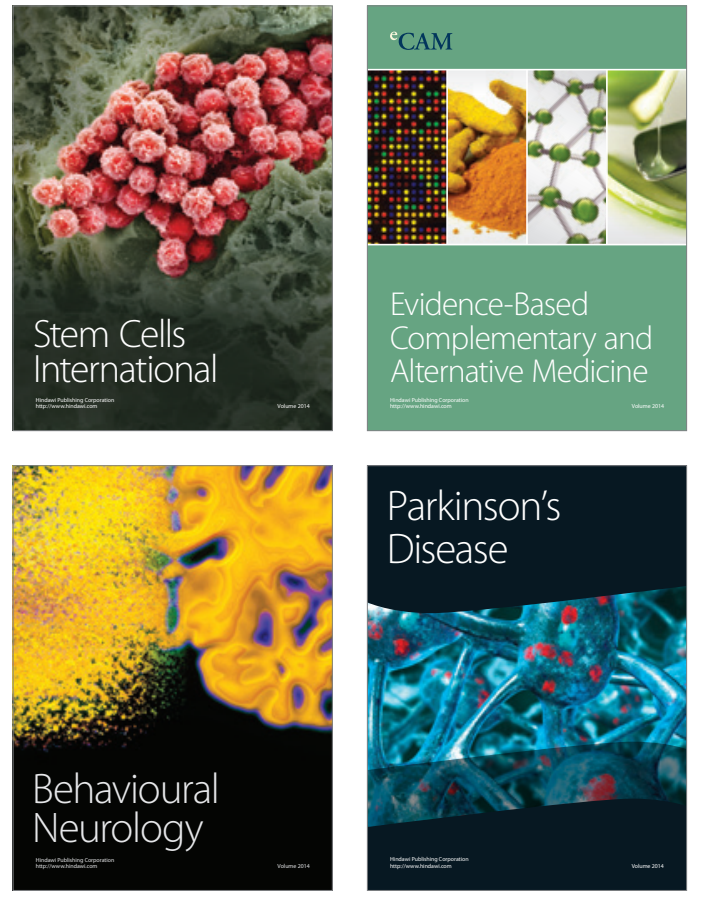
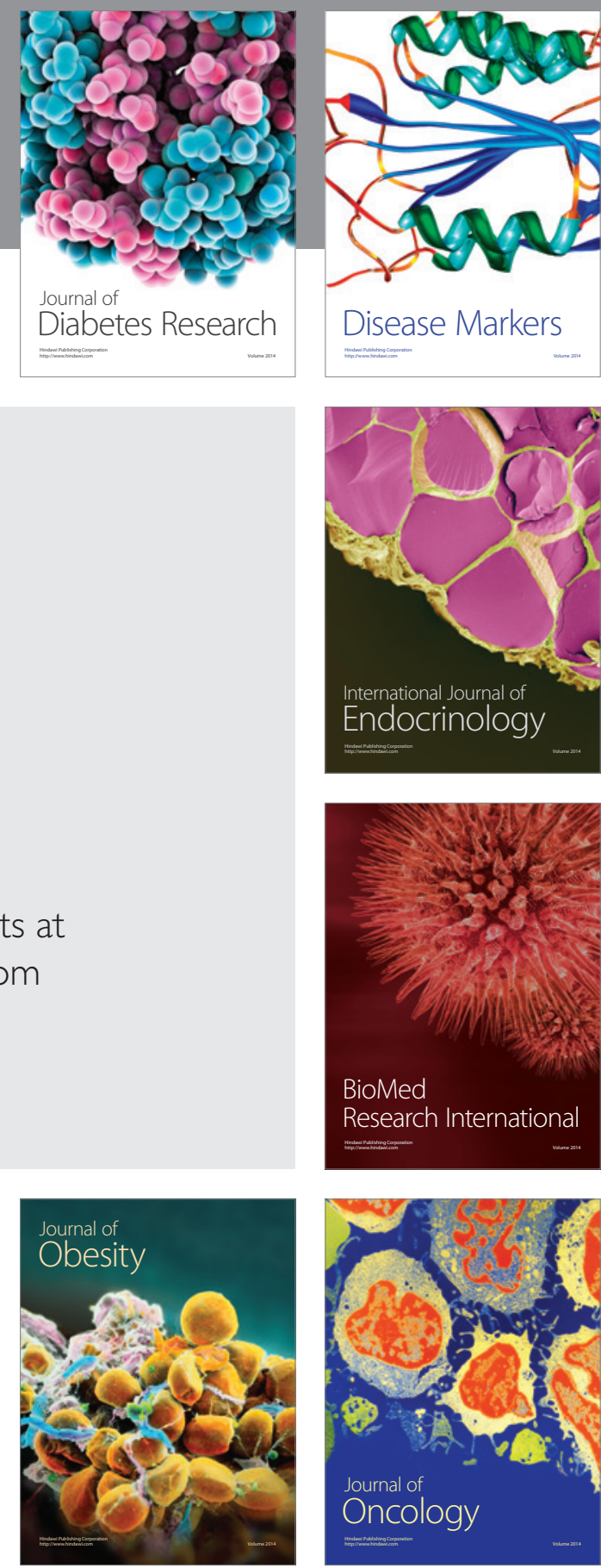

Disease Markers
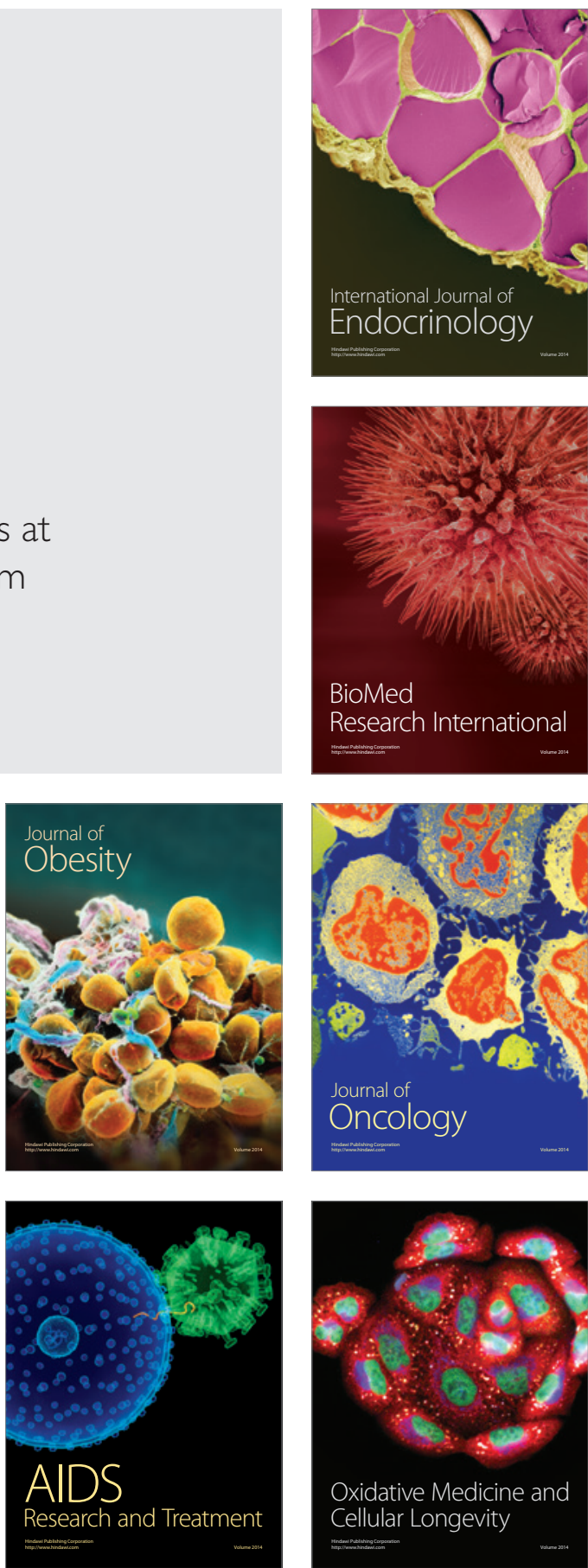